\title{
Labyrinthe
}

$6 \mid 2000$

Numéro 6

Recherche

\section{Transition économique et contrainte de budget : l'exemple bulgare}

\section{Émeric Uhring}

\section{(2) OpenEdition \\ Journals}

\section{Édition électronique}

URL : http://journals.openedition.org/labyrinthe/435

DOI : $10.4000 /$ labyrinthe.435

ISSN : 1950-6031

\section{Éditeur}

Hermann

\section{Édition imprimée}

Date de publication : 1 juin 2000

Pagination : 155-161

\section{Référence électronique}

Émeric Uhring, «Transition économique et contrainte de budget : l'exemple bulgare », Labyrinthe [En ligne], 6 | 2000, mis en ligne le 23 mars 2005, consulté le 24 avril 2019. URL : http:// journals.openedition.org/labyrinthe/435 ; DOI : 10.4000/labyrinthe.435

Ce document a été généré automatiquement le 24 avril 2019.

Propriété intellectuelle 


\title{
Recherche
}

\section{Transition économique et contrainte de budget : l'exemple bulgare}

\author{
Émeric Uhring
}

Synthèse d'un mémoire de D.E.A. d'économie appliquée préparé à l'IEP de Paris sous la direction de Gérard Wild et Patrick Messerlin, et soutenu en septembre 1999.

1 Dix ans après les débuts de la transition qui doit les intégrer, économiquement et politiquement, au reste du continent, les différents pays d'Europe centrale et orientale ont obtenu des résultats parfois très divergents. Quel rapport entre la situation en Hongrie, de plus en plus proche de l'Union Européenne, et celle en Roumanie, pourtant voisine ? Les conditions économiques et structurelles initiales ont joué leur rôle. Mais il serait trop simple de rejeter la responsabilité des difficultés présentes sur les errements d'un passé révolu. Des erreurs ont été commises. Le mauvais gouvernement n'est évidemment pas la seule cause du retard de certains pays. Pourtant, à mesure que le temps passe et que se révèlent les résultats obtenus par les diverses méthodes de transition, il se dégage de plus en plus nettement que la qualité de la gouvernance a pesé d'un grand poids dans l'avancement et la réussite des réformes. Jeter la pierre à ceux qui ont échoué peut aujourd'hui paraître facile, car au départ, tous ont commencé par tâtonner.

2 La transition vers le marché est en effet un phénomène nouveau dans la liste des questions posées à l'économie. Son originalité réside dans le fait que, au début du processus, les pays concernés étaient largement industrialisés et sociologiquement proches des membres de l'OCDE mais présentaient un retard très important en termes de revenu par habitant et un décalage tout aussi conséquent dans le poids respectif des différents secteurs d'activité dans l'économie. La tâche de leurs gouvernements était par conséquent de promouvoir une large réorganisation des structures économiques afin de 
rendre possible le rattrapage des niveaux de production et, surtout, de vie. La chute des régimes dits " socialistes " a en grande partie tenu à leur incapacité à assurer à leurs populations un confort comparable à celui dont jouissent les habitants des pays capitalistes développés. "L'objectif global de la transition [était] donc de promouvoir la croissance ${ }^{1}$. " Et la tâche apparaissait d'emblée très importante. En effet, même en maintenant constamment un taux de croissance du PIB aux alentours de 6-7 \% par an, les pays d'Europe centrale et orientale ne pourraient rejoindre la moyenne des pays de l'OCDE qu'au bout de 20 à 40 ans (Anderson, Berglöf \& Miszei, 1996). Encore ces calculs ne tiennent-ils pas compte des difficultés inhérentes au processus et qui perturbent plus ou moins gravement le fonctionnement des structures économiques héritées de la période précédente. En effet, adaptées à une économie planifiée, elles se sont révélées difficiles à conformer aux nécessités du marché. Pour les gouvernements, il a fallu faire face avec plus ou moins de bonheur à des difficultés à la fois macro- et micro-économiques.

3 Au début de la transition, la principale question qui se posait aux nouvelles équipes gouvernementales était la suivante : par quoi commencer ? La réponse des experts occidentaux appelés en renfort a tenu en deux mots, libéralisation et stabilisation, deux concepts sur lesquels le consensus était très large. Les gouvernements ont dû provoquer et tenter d'accompagner une transformation profonde des relations de l'État avec les autres agents économiques, qui auparavant dépendaient largement des impulsions venues $\mathrm{du}$ centre. Cette rupture micro-économique a eu de profondes répercussions macro-économiques. L'État s'est trouvé simultanément confronté à une série de déséquilibres majeurs qu'il lui a fallu corriger au plus vite : très forte inflation, déficits commercial et courant, déficit budgétaire, apparition du chômage... Aussi une économie en transition doit-elle passer par une phase de stabilisation macro-économique, sur laquelle l'accent a été mis dans les premières années de la transition.

4 Mais le gouvernement se trouve également chargé d'un secteur public omniprésent et obsolète, dont la restructuration et la privatisation sont indispensables au rétablissement des grands équilibres. Il s'agit là d'une tâche qui non seulement absorbe une grande partie de l'énergie des administrations mais qui implique parfois aussi des mesures énergiques. Et ces dernières peuvent se trouver en contradiction avec les priorités affichées par ailleurs par les gouvernements (maintien de l'emploi, priorité donnée aux investisseurs locaux...). Aussi la restructuration des firmes publiques a-t-elle dans certains cas été ralentie, voire différée. En outre, les gouvernements démocratiques ont dû reconstruire un État déconsidéré après la chute du régime précédent, ce qui peut en partie expliquer le manque de vigueur parfois constaté de leurs politiques. Or comme le souligne Yang Haiqun, " la perte de contrôle du pouvoir central sur les entreprises conduit de désordres micro-économiques en chaos macro-économique ${ }^{2} »$. Et c'est bien là le nœud du problème, car cet aspect a longtemps été négligé par la recherche sur la transition, occulté par les grands débats sur la priorité à donner à une « thérapie de choc " à la polonaise ou une approche graduelle à la hongroise. La micro-économie est pourtant au cœur des perfectionnements théoriques de la macro-économie depuis la fin des années 70. Les crises de la deuxième moitié des années 90 ont enfin permis d'attirer l'attention des analystes sur l'importance des réformes micro-économiques.

5 Passé le premier choc de la libéralisation, qui avait logiquement entraîné un effondrement de la production, les pays d'Europe centrale et orientale (PECO) semblaient dès 1994 avoir surmonté cette épreuve. Les taux de croissance étaient partout positifs et paraissaient devoir s'accélérer afin de permettre le rattrapage attendu avec l'UE. Or dès 
1996 la Bulgarie plongeait dans une crise sévère. Depuis, d'autres PECO ont connu ou connaissent encore une seconde période de récession plus ou moins marquée alors que certains d'entre eux, comme la République tchèque, étaient encore récemment présentés comme des modèles. De plus, tous avaient mis en place des plans de stabilisation macroéconomiques avec l'aide des institutions financières internationales. La réflexion s'est alors emparée de ces événements pour comprendre pourquoi et comment la transition aboutissait à des résultats aussi tranchés : les réussites comme les échecs ne semblaient pouvoir être que flagrants.

6 Ma recherche s'est focalisée sur le système de caisse d'émission ${ }^{3}$ mis en place en Bulgarie après l'effondrement économique et monétaire survenu en 1996 et au début de l'année 1997. Le CBA est un système qui, en adossant strictement la monnaie nationale, selon un taux fixe, à une monnaie de référence, prive le pays qui en fait le choix de toute souveraineté en matière de politique monétaire mais est censé lui offrir en échange la stabilité qui règne dans le pays de la monnaie de référence. Il connaît un regain d'intérêt depuis le début des années 90, en particulier dans les pays confrontés à de graves problèmes inflationnistes. L'Argentine a ouvert la voie, même si la théorie des CBA conseille plutôt d'appliquer ce genre de solution à des petits pays largement ouverts aux flux internationaux. Mais c'est à propos des pays en transition que le débat a été le plus animé. L'un des conseillers occidentaux les plus écoutés, Steve Hanke ${ }^{4}$, est en effet un fervent apôtre de ce système comme moyen de stabilisation et il a suscité un débat très animé, et très anglo-saxon, autour de ce sujet. Mon premier mouvement a donc été de poser les termes de ce débat et de discuter de la pertinence du choix d'un tel arrangement pour la Bulgarie. J'en ai conclu que l'état de son économie, et notamment l'exacerbation du phénomène de fuite devant la monnaie nationale (le lev), ne lui en laissait guère d'autre.

7 J'ai toutefois rapidement été amené à en dépasser les aspects purement macroéconomiques. En effet, par la stricte orthodoxie en matière de déficits extérieurs et budgétaires que réclame le fonctionnement d'un $\mathrm{CBA}$, ce dernier exige une discipline financière tout aussi rigoureuse de la part de l'ensemble des agents économiques du pays. Or il ressort des études menées depuis 1996 que les difficultés de la plupart des PECO dont la transition a été chaotique sont dues pour une grande part la persistance dans une économie capitaliste de la « contrainte de budget molle ${ }^{5}$ " qui aurait caractérisé les économies socialistes. En d'autres termes, les entreprises structurellement déficitaires n'étaient pas sanctionnées par la faillite mais recevaient au contraire subventions et prêts bancaires leur permettant de survivre. Les exigences des plans de stabilisation du début des années 90 ont progressivement fait disparaître les subventions à ces entreprises dans la plupart des PECO. Restaient les prêts bancaires et c'est sur ce point précis que s'est fait le départ entre pays performants et pays voués à l'échec. Ceux qui ont rapidement soustrait les établissements bancaires aux influences politiques et industrielles et qui ont mis en place une législation et une supervision efficaces, calquées sur les standards internationaux, ont ainsi créé les conditions du fonctionnement normal d'une économie capitaliste. En revanche, les pays qui, à l'instar de la Bulgarie, ont négligé ce point, ont permis à un volume énorme de créances douteuses de s'accumuler dans l'économie, jusqu'à ce que des déséquilibres majeurs fragilisent, voire emportent, à la fois le système financier et la monnaie et provoquent une nouvelle récession. Ainsi, le contre-exemple bulgare illustre à merveille la nécessité de conduire simultanément la stabilisation macro-économique et les réformes micro-économiques, à savoir modernisation du 
système bancaire, mais aussi législatif et judiciaire, ainsi que restructuration des entreprises industrielles.

Ce caractère global des réformes à entreprendre a été souligné par les travaux menés par Andrew Berg, e.a. (1999) ${ }^{6}$ au sein du FMI. Dans le cadre d'une étude générale portant sur 26 pays en transition (de la Pologne à la Mongolie), ces études se sont attachées à isoler les déterminants essentiels expliquant les différences dans la reprise de la croissance entre tous ces pays. Or ce qui est ressorti clairement de leurs recherches, c'est que tant les conditions initiales de la transition que les variables macro-économiques, qui n'apparaissent que comme un préalable, ne jouaient pas un rôle prééminent dans la reprise de la croissance du PIB. La force motrice derrière la reprise d'une croissance soutenue serait, selon cette étude - l'exemple bulgare semble corroborer ce point -, la vigueur, mais aussi la rapidité des réformes structurelles entreprises. Voilà qui apporte des arguments en faveur de la "thérapie de choc ", à condition toutefois qu'elle ne se limite pas à la macro-économie. Le règlement des problèmes de mise en place des mécanismes de marché (information, régulation...) constitue à ce titre un point particulièrement important ${ }^{7}$. La conclusion de ma recherche a été que, dans le cas d'un pays ayant comme la Bulgarie manqué les débuts de sa transition, un système de caisse d'émission se prête avec plus de facilité à l'imposition d'un tel bouleversement, à la fois micro- et macro-économique, qu'un système de banque centrale.

9 Le débat économique sur la transition est malheureusement très pauvre en France et reflète la faible présence française dans les PECO. La plupart des études sont menées par des centres de recherches anglo-saxons, voire germaniques, ou des institutions financières internationales.

\section{BIBLIOGRAPHIE}

ANDERSON Ronald \& KEGELS Chantal, Transition Banking : Financial Developement of Central and Eastern Europe, Oxford University Press, 1998, 300 p.

BALIÑO Tomás \& ENOCH Charles, Currency Boards arrangements : issues and experiences, International Monetary Fund, Washington DC, 1997, 52 p.

BERG Andrew, BORENSZTEIN Eduardo, SAHAY Ratna \& ZETTELMAYER Jeromin, The Evolution of Outpout in Transition Economies : Explaining the Differences, IMF Working Paper nº 99/73, may 1999, $81 \mathrm{p}$.

BERGLÖF Erik \& ROLAND Gérard, « Soft Budget Constraint and Banking in Transition Economies », dans Journal of Comparative Economics, 1998-03, vol. 26 n 1, p. 18-40.

BLANCHARD Olivier, The Economics of Post-Communist Transition, Clarendon Press, Oxford, 1997, 149 p.

BLEJER Mario \& SKREB Marko (ed.), Macroeconomic Stabilization in Transition Economies, Cambridge University Press, 1997, 338 p.

GHOSH Atish R., GULDE Anne-Marie, and wolf Holger C., Currency Boards : The Ultimate Fix ?, IMF Working Paper 98/8, 1998, 22 p. 
HANKE Steve, «Currency Boards : la thérapie oubliée », dans Politique Internationale, n 80, été 1998, p. 25-39.

HANKE Steve, « Monetary Stability for Economics in Transition », dans Zagreb Journal of Economics, $\mathrm{n}^{\circ} 3,1999$, p. 23-50.

KoRNAI János, «The Place of the Soft Budget Constraint Syndrome in Economic Theory », dans Journal of Comparative Economics, 1998-03, vol. 26 n ¹, p. 11-17.

ROSTOWSKI Jacek, Macroeconomic instability in post-communist countries, Oxford University Press, $1998,380 \mathrm{p}$.

SCHULER Kurt, Should Developing Countries Have Central Banks ?, Research Monograph $n^{\circ} 52$, Institute of Economic Affairs, London, 1996, 126 p.

SCHWARTZ Anna, Do Currency Boards have a Future?, Institute of Economic Affairs, London, 1992, 24 p.

SGARD Jérôme et AVRAMOV Roumen, Bulgaria : From Enterprise Indiscipline to Financial Crisis, CEPII document de travail n 96-10, 1996, $42 \mathrm{p}$.

WILLIAMSON John, What Role for Currency Boards?, Institute for International Economics, Washington DC, 1995, 44 p.

Il faut ajouter à cette liste les sites internet du FMI (www.imf.org), de la Banque Mondiale (www.worldbank. org), de la Banque centrale de Bulgarie (www.bnb.bg), où l'on peut trouver de nombreux papiers sur les caisses d'émission et les économies en transition, ainsi que le site de Kurt Schuler consacré aux CBA (http ://users. erols. com/kurrency).

\section{NOTES}

1. «Thus the broad objective of transition is to promote growth », dans Ronald W. Anderson, Erik Berglöf, Kálmán Miszei, Banking Sector Development in Central and Eastern Europe, 1996, p. 1.

2.«Losing central control over firms leads from microeconomic disorders into macroeconomic chaos », dans Yang Haiqun, « Control or Decontrol ? Theoretical arguments for policy making in transition ", International Review of Applied Economics, vol. 10, $\mathrm{n}^{\circ} 2,1996$, p. 228.

3. Currency Board Arrangement ou CBA en anglais.

4.Professeur à la Johns Hopkins University, conseiller de plusieurs gouvernements dont la Bulgarie, l'Estonie et le Monténégro. Cf. bibliographie.

5.Ou soft budget constraint, principe exposé dès 1979 par l'économiste hongrois Janos Kornai (cf. bibliographie).

6.Cf. bibliographie.

7.Cf. STIGLITZ Joseph, Knowledge for Development : Economic Science, Economic Policy and Economic Advice, Address to the World Bank's 10 Annual Bank Conference on Development Economics (ABCDE), April 20, 1998. 


\section{AUTEUR}

\section{ÉMERIC UHRING}

emeric.uhring(at)wanadoo.fr 